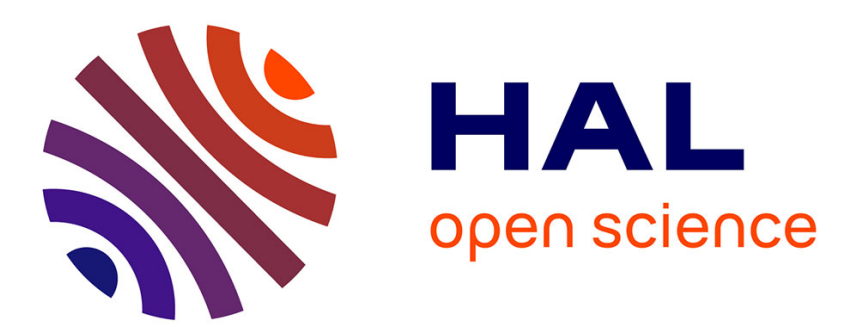

\title{
Analysis of the fault injection mechanism related to negative and positive power supply glitches using an on-chip voltmeter
}

Loic Zussa, Jean-Max Dutertre, Jessy Clediere, Bruno Robisson

\section{- To cite this version:}

Loic Zussa, Jean-Max Dutertre, Jessy Clediere, Bruno Robisson. Analysis of the fault injection mechanism related to negative and positive power supply glitches using an on-chip voltmeter. IEEE Int. Symposium on Hardware-Oriented Security and Trust (HOST), May 2014, Arlington, France. 10.1109/HST.2014.6855583 . emse-01099010

\section{HAL Id: emse-01099010 \\ https://hal-emse.ccsd.cnrs.fr/emse-01099010}

Submitted on 30 Dec 2014

HAL is a multi-disciplinary open access archive for the deposit and dissemination of scientific research documents, whether they are published or not. The documents may come from teaching and research institutions in France or abroad, or from public or private research centers.
L'archive ouverte pluridisciplinaire HAL, est destinée au dépôt et à la diffusion de documents scientifiques de niveau recherche, publiés ou non, émanant des établissements d'enseignement et de recherche français ou étrangers, des laboratoires publics ou privés. 


\title{
Analysis of the fault injection mechanism related to negative and positive power supply glitches using an on-chip voltmeter
}

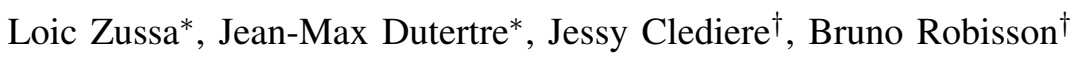 \\ ${ }^{*}$ Ecole Nationale Superieure des Mines de Saint-Etienne (ENSM.SE) \\ Gardanne, France \\ Email: \{loic.zussa,jean-max.dutertre\}@ mines-stetienne.fr \\ ${ }^{\dagger}$ Commissariat a l'Energie Atomique et aux Energies Alternatives (CEA) \\ Gardanne/Grenoble, France \\ Email: \{bruno.robisson,jessy.clediere $\}$ cea.fr
}

doi: 10.1109/HST.2014.6855583 


\begin{abstract}
Power supply underpowering and negative power supply glitches are commonly used for the purpose of injecting faults into secure circuits. The related fault injection mechanism has been extensively studied: it is based on setup time violations. Positive power supply glitches are also used to inject faults. However, an increase of the supply voltage is not consistent with a mechanism based on setup time violation. Besides, no research work has yet identified the corresponding mechanism. In this work, we report the use of an embedded delay-meter to monitor the core voltage of a programmable device exposed to power supply glitches. It permitted us to gain a further insight into the mechanism associated with power glitches and also to identify the injection mechanism of positive power supply glitches.
\end{abstract}

\section{INTRODUCTION}

Secure circuits are targeted by a wide range of physical attacks. Among these are fault attacks (FA) based on modifying the circuit environment in order to change its behaviour or to induce faults into its computations. Fault injection (FI) may be performed thanks to several means: laser shots, overclocking, underpowering, temperature increase, power supply glitches or electromagnetic pulses. A fine understanding of the properties and the mechanisms involved with these FI techniques is of high interest to evaluate the related threats and also to facilitate the design of counter-measures.

Laser FI has a specific mechanism that is well known. Whereas FI based on setup time violations [1], [2] may be obtained by various techniques: overclocking , underpowering [1], temperature increase, clock and negative power supply glitches, and to a certain extent EM pulses. The latter FI techniques are linked to an increase of the target's propagation delays over its clock period (see section III).

Positive power supply glitches, despite being a practical fault injection means [3], have been less studied. It can not be explained by a setup time violation because a voltage increase will lead to a decrease of the target's propagation times, which is inconsistent with this hypothesis. Its FI mechanism has not yet been ascertained.

In this paper, we report the use of a digital delay-meter embedded in a programmable device (FPGA) as presented in [4] and [5] for the purpose of identifying the fault injection mechanism related to positive power supply glitches. It allowed us to monitor the core voltage of the device when exposed to positive and negative power supply glitches. It also made it possible to correlate the perturbations induced on the target's core voltage with the faults injected into an implementation of the advanced encryption standard (AES [6]). Our contributions to that research field are:

- An illustration of the use of a delay-meter to monitor the core voltage of a circuit exposed to power supply glitches,

- An identification of the fault injection mechanism related to positive power supply glitches,

- An in-depth experimental study of the practice of FI with power supply glitches.

This article is organized as follows. In section II a reminder of timing constraints and an explanation of how faults may be injected by their violation is provided. In section III the architecture and principles of the delay-meter used as an on-chip voltmeter are described. The experimental setup is described in section IV The obtained experimental results are presented and analyzed in section $\mathrm{V}$. Finally, our findings are summarized in section VI with some perspectives.

\section{PRELIMINARIES}

In this section the basics of timing constraints are summarized. Then the mechanism of fault injection through timing constraint violations is reviewed.

\section{A. Timing constraints}

Almost all digital ICs use a common clock signal to synchronize their internal operations. Fig. 1 illustrates the corresponding architecture. Data are computed by combinatorial logic (marked $\sum$ ) surrounded upstream and downstream by register banks made of D flip-flops (DFF) sharing the same clock signal $(c l k)$.

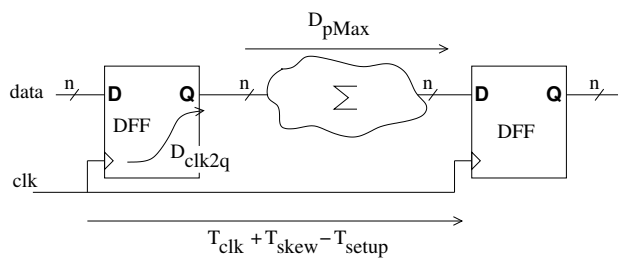

Fig. 1. Internal architecture of digital ICs.

On a clock rising edge, data are released from the upstream DFF after a delay $D_{c l k 2 q}$ which represents the delay between the clock rising edge and the actual update of a register's output. Then, these data are processed through the logic before being latched into the next register on the next clock rising edge. The data propagation time, $D_{p(\text { data })}$, through the logic is not constant. It depends on the handled data (those of the current and previous clock periods) and also on the power supply voltage. $D_{p M a x}$ is the longest propagation time (i.e. the logic critical time).

A proper functioning of a DFF requires its input to be stable all along a time window that extends before and after the clock rising edge from the setup $\left(T_{\text {setup }}\right)$ and hold $\left(T_{\text {hold }}\right)$ times respectively. The data shall not arrive too late nor too early. This arrival constraints can be described mathematically with two timing constraint equations: the setup time timing constraint expressed in eq. 1 and the hold time timing constraint expressed in eq. 2] [7].

$$
\begin{gathered}
T_{c l k}>D_{c l k 2 q}+D_{p M a x}+T_{\text {setup }}-T_{\text {skew }} \\
T_{\text {hold }}<D_{\text {clk } 2 q}+D_{p M i n}+T_{\text {skew }}
\end{gathered}
$$

where $T_{\text {skew }}$ is the slight phase difference that may exist between the clock signals at the clock inputs of two different registers due to the clock propagation times through the clock network, and $D_{p M i n}$ is the minimal propagation time through the logic. 


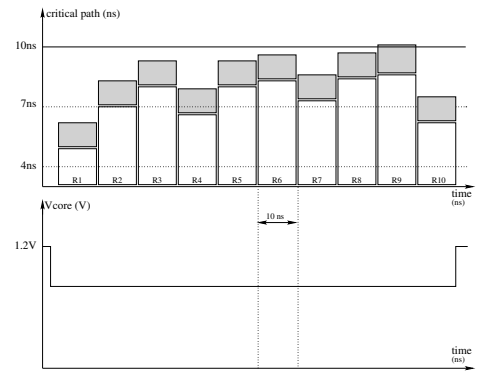

(a)

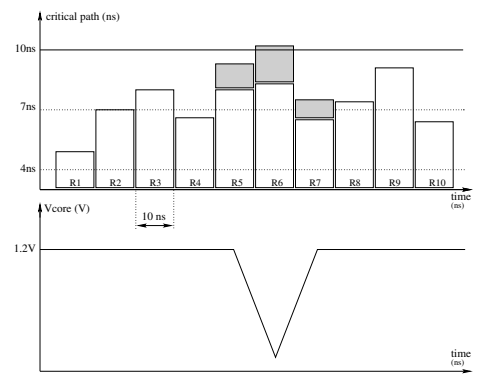

(b)

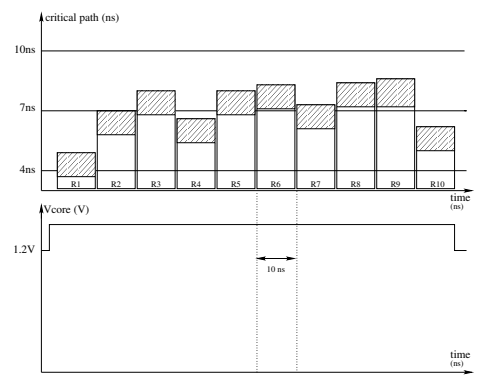

(c)

Fig. 2. Critical paths of the AES' rounds when subject to: (a) underpowering, (b) a negative power supply glitch, (c) overpowering.

\section{B. Fault injection due to timing constraints violations}

The violation of these timing constraints is a means to inject faults into synchronous integrated circuits (IC).

Previous studies have shown how underpowering a synchronous circuit leads to setup time violations [1], [2], [8]. As the target's supply voltage is decreased, the propagation times through its logic are increased. Consequently, a violation of the setup time constraint may happen when the propagation time increase is large enough: thus a fault is induced. Fig. 2(a) illustrates the effect of underpowering an IC implementing the AES algorithm (its nominal voltage and clock period are $1.2 \mathrm{~V}$ and $10 \mathrm{~ns}$ respectively). The critical times of the AES' rounds at nominal voltage are depicted in white on the upper curve (they are obviously shorter than the clock period in order to comply with the setup time constraint). The bottom curve represents the decrease of the target's core voltage (i.e. the underpowering). As a result, the critical times of the whole AES' rounds are increased (depicted in grey). In this illustration a setup time violation occurs during the $9^{\text {th }}$ round.

Fig. 2(b) illustrates the effect of a negative power supply glitch: the critical time of the round centered on the glitch (and also those of its neighbors rounds) is increased. As a result, the setup time violation occurs during the $6^{\text {th }}$ round. This allows an attacker to target a given round, which is mandatory to perform a successful differential fault attack [9].

Fig. 2(c) illustrates the effect of an increase of the target's core voltage (i.e. overpowering): its critical times are decreased (depicted with grey stripes). No faults were obtained because there were neither setup time nor hold time violations (for the latter, the rounds' propagation times are too large). Note that the illustrations of Fig. 2 are based on actual experiments. We were not able to inject faults into the AES' computations by overpowering. However, faults may be injected in the parts an IC which has very small propagation times: e.g. a shift register.

Nevertheless, we have successfully injected faults into the AES' computations using positive power supply glitches. According to the previous examples, the related FI mechanism seemed unlikely to be due to setup or hold time violations. It was the main motivation of our work: sensing the core voltage of an IC when exposed to voltage glitches in order to find out the relevant FI mechanism.

\section{Voltmeter Design}

Previous experiments [2] on the IC used to carry out the experimental part of this paper showed that the propagation times of the IC logic vary linearly with its power supply voltage. Consequently, measuring the delay of a logic block reveals its supply voltage value. Thus, for the purpose of monitoring the core voltage of an IC exposed to power glitches, we designed a delay-meter (the so-called voltmeter). Note that this technique has already been introduced and validated by K. Zick et al. [4].

Fig. 3 shows the simplified architecture of the delay-meter. It is made out of two blocks:

- a delay block for which the delay depends on the IC's core voltage,

- a time-to-digital converter (TDC [10]) used to obtain a binary code correlated with the delay.

The input of the delay-meter is the clock signal of the device $(c l k)$. It is fed into the delay block to produce a delayed clock, $c l k_{\text {delay }(0)}$. The corresponding delay, delay $(V d d)$, depends on its supply voltage $V d d$. Then, the TDC is used to convert the phase difference between $c l k$ and $c l k_{\text {delay }(0)}$ into a binary code. The TDC consists of a series of 8 delay elements (their elementary delay is $\delta d$ ). Its input is the delayed clock $c l k_{\text {delay }(0)}$. Thus, it provides 8 delayed clocks with an additional delay: $n * \delta d$, where $\mathrm{n}$ is the index of the corresponding delay element. Then, 8 DFFs are used to compare the phases of the delayed clocks with the main clock $c l k$. The output of the $n^{\text {th }}$ DFF is low, resp. high, when $c l k_{\operatorname{delay}(n)}$ is in phase advance, resp. in phase delay, with respect to $c l k$. As a result, the outputs of the DFFs form an 8-bit vector, which depends on delay $(V d d)$ ([4], [10]). The right-hand side of Fig. 3 illustrates the obtained output vectors for different settings of the core voltage. As $V d d$ varies, the phase shift between the clock signals also varies leading to a change in the TDC's output vector. The obtained binary code is a thermometer code: it consists in 2 blocks of consecutive " 0 " and " 1 ". The information it contains is twofold:

- its Hamming Weight (HW),

- the order of these blocks to differentiate e.g. "000111" and "111000".

Thus, to take into account both these pieces of information, we used the notion of Signed Hamming Weight (SHW): e.g. 


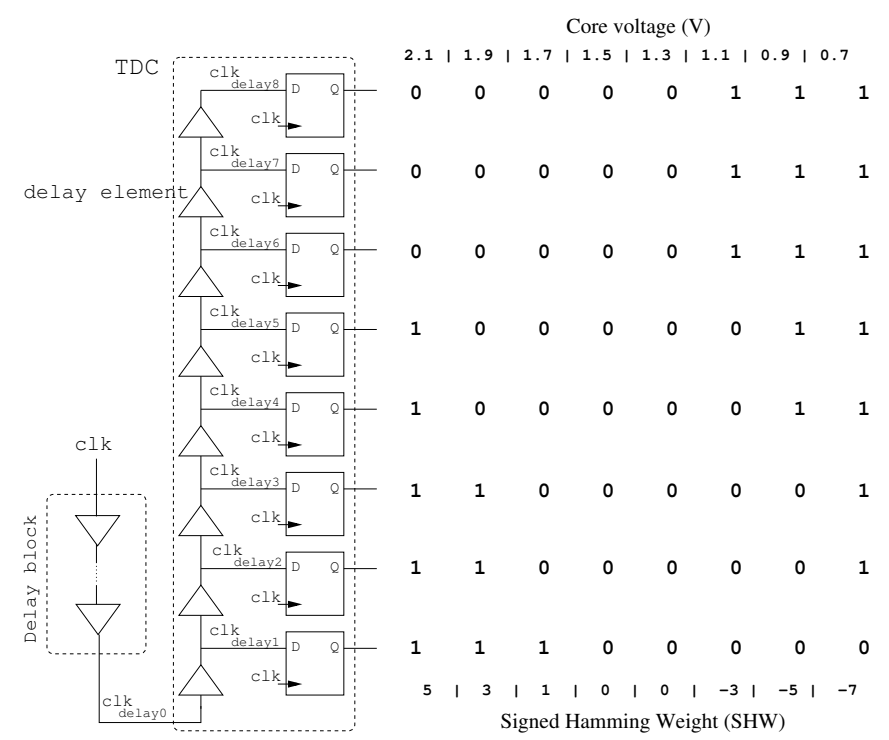

Fig. 3. Simplified architecture of the delay-meter (i.e. voltmeter).

"00000111" and "11100000" were respectively denoted " +3 " and "-3" (see also Fig. 3).

We used 4 instances of the previous delay-meter (with different delay blocks) to build a voltmeter with a proper voltage measurement range and resolution: $0.7 \mathrm{~V}$ to $2.4 \mathrm{~V}$ and around $20 \mathrm{mV}$ respectively. Note that the voltage resolution was not constant over the whole measurement range. Fig. 4 displays its SHW output as a function of the core voltage. The device embedding the voltmeter was exposed to static voltage underpowering to gather the measures used to build this curve. The bijective function corresponding to this curve was used

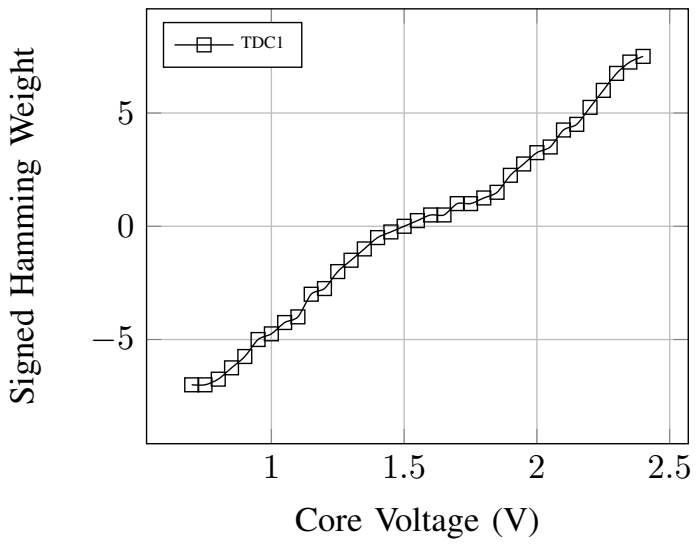

Fig. 4. TDC outputs vs. core voltage.

hereafter to convert the output vectors of the voltmeter into a voltage; despite the fact that the corresponding experiments were carried out with dynamic perturbations (i.e. glitches).

\section{EXPERIMENTAL SETUP}

In this study we used two different setups, a voltmeter and an AES, embedded alternately in the same test vehicle: a programmable circuit (FPGA - Xilinx Spartan3 700A).

\section{A. Pulse generator}

We used an Agilent 8114A pulse generator to inject power supply glitches into our test vehicle. This pulse generator is able to produce positive or negative voltage pulses in the $1 \mathrm{~V}$ to $50 \mathrm{~V}$ range, with constant fall and rise times equal to $10 \mathrm{~ns}$ and a tunable pulse width between $10 \mathrm{~ns}$ and $100 \mathrm{~ms}$. The voltage pulse is centered on a tunable DC voltage. We used this feature to provide the voltage supply of the test chip and also to set and modify at will its value.

\section{B. Voltmeter setup}

This subsection presents the first FPGA setup used to sense its core voltage when exposed to power supply glitches. The voltmeter introduced in section $\amalg$ III is well suited to measure a static voltage. Its output is updated at the rate of its clock signal. Its period is $5 \mathrm{~ns}$. In order to monitor a transient perturbation (i.e. a voltage glitch), a 30 stage shift register (SR) with an 8-bit width was connected to its output. It makes it possible to register the core voltage over a $150 \mathrm{~ns}$ range with a 5 ns time resolution (it was not feasible to further increase the length of the SR because of design and practical constraints).

Because the FPGA and the pulse generator were synchronized thanks to a common trigger signal, we were able to extend this time window by carrying out the measurements at different times for identical settings (i.e. the voltage pulse parameters). The measurements were carried out over a global time range of $750 \mathrm{~ns}$ by dividing it in time slots of $150 \mathrm{~ns}$. Moreover, during each time slot the experiments were reproduced 5 times with a successive time increment of $1 \mathrm{~ns}$. Hence, the time resolution was upgraded to $1 \mathrm{~ns}$. In addition, all these measurements were conducted three times and then averaged.

Note also that, many of the voltage glitch injection experiments were performed with a core voltage set over the FPGA nominal voltage of $1.2 \mathrm{~V}$. In order to center the glitch within the voltmeter's best achievable resolution range and thus to obtain accurate measurements (this was the case for the curves displayed in section $\mathrm{V}$.

\section{Fault injection setup}

This subsection presents the second FPGA setup used to inject faults and verify our assumptions about the fault injection mechanisms. The target is a hardware 128-bit AES designed to operate at a 10ns clock period and to complete a whole encryption in 11 clock cycles. In this work, the 128-bit AES ([6]) is mainly used as a test element. Thus, we will not go deeper into its properties. However, because this algorithm is likely to be subject to FA, the obtained results are still of interest.

We used the following methodology to induce faults into the AES' calculations: we kept constant the pulse amplitude (either positive or negative) and width settings while varying both the core voltage provided by the pulse generator to the test chip and the starting time of the pulse. At first, the core voltage was set over its nominal voltage and then progressively 
decreased until a first fault was induced. The starting time of the pulse was also swept along a time range encompassing the whole AES in order to target all its rounds. Then, other pulse amplitude and width settings were tested according to the same methodology.

\section{EXPERIMENTAL RESULTS}

First, we performed both negative and positive power supply glitches with a pulse width varying from 10ns to 500ns and an amplitude varying from $1 \mathrm{~V}$ to $50 \mathrm{~V}$. During these injections the core voltage of the FPGA was observed with the on-chip voltmeter. Then, these obtained waveforms were analyzed and an assumption about the fault injection mechanism related to positive power supply glitches was made. Second, we verified this assumption by targeting an AES with relevant positive and negative power supply glitches.

\section{A. Negative power supply glitch effects on the core voltage}

1) Observations: The first injection experiments with negative voltage glitches were carried out on the voltmeter. We observed two sets of damping oscillations corresponding to the falling and rising edges of the injected voltage pulse. As an example, Fig. 5 displays the FPGA core voltage (measured with the on-chip voltmeter) for a voltage pulse of $-14 \mathrm{~V}$ amplitude and 400ns duration. The time interval between the 2 sets of damping oscillations is $400 \mathrm{~ns}$, which is also the time interval between the falling and rising edges of the power glitch. The first negative oscillation after the perturbation's falling edge is the biggest: its amplitude is close to $400 \mathrm{mV}$ and its width at the oscillation's tip is around 20ns. Similar shapes for the core

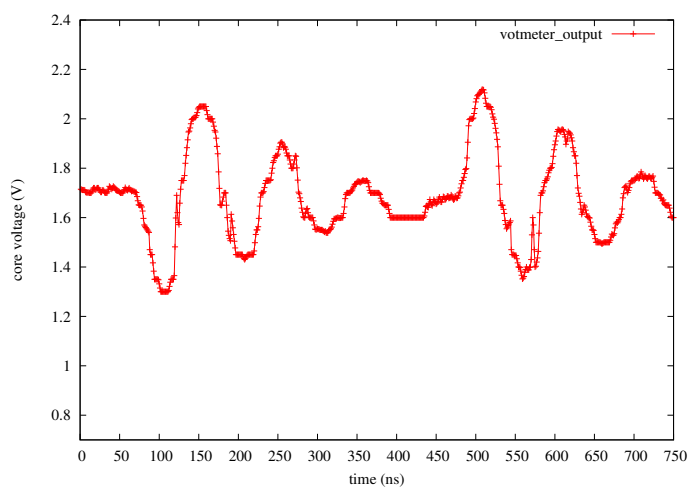

Fig. 5. Core voltage of the FPGA for a (400ns, -14V) power supply glitch.

voltage were measured for other settings of the pulse's parameters. Analyzing the obtained core voltage shapes, we drew the assumption that the first negative oscillation following the pulse's falling edge was well suited to induce faults into an IC: it might be used according to the principle depicted in Fig. 2(b) Fault injection is a result of setup time violations induced by a transient underpowering of the target corresponding to the oscillation width.
2) Fault injection: Consequently, the $2^{\text {nd }}$ set of experiments was performed on the AES setup (see subsection IV-C) with a $(400 \mathrm{~ns},-14 \mathrm{~V})$ pulse. The moment of the negative oscillation's tip was swept over the AES' rounds. Moreover, for each time location, the DC component of the glitch was gradually decreased from $1.7 \mathrm{~V}$ until the injection of the first faults. As a result, the voltage of the oscillation tip went under the target's nominal voltage $(1.2 \mathrm{~V})$ inducing a setup time violation.

This methodology made it possible to inject faults into every AES round (except the first one). We also verified that the faulted rounds always corresponded with the time location of the oscillation tip. Most of the induced faults were single-bit faults affecting only one round. For the sake of brevity, these results are not detailed here. However, subsection V-C4 reports very similar results.

\section{B. Positive power supply glitch effects on the core voltage}

1) Observations: Fig. 6 displays the FPGA's core voltage measured with the voltmeter when exposed to a positive power supply glitch. Its duration and amplitude were 400ns and $+14 \mathrm{~V}$ respectively. The induced core voltage perturbation is very similar to that induced by a negative voltage pulse (see Fig. 5), except that the damping oscillation sets corresponding to the falling and rising edges are inverted. Similar shapes were obtained with close settings of the voltage pulse. The main

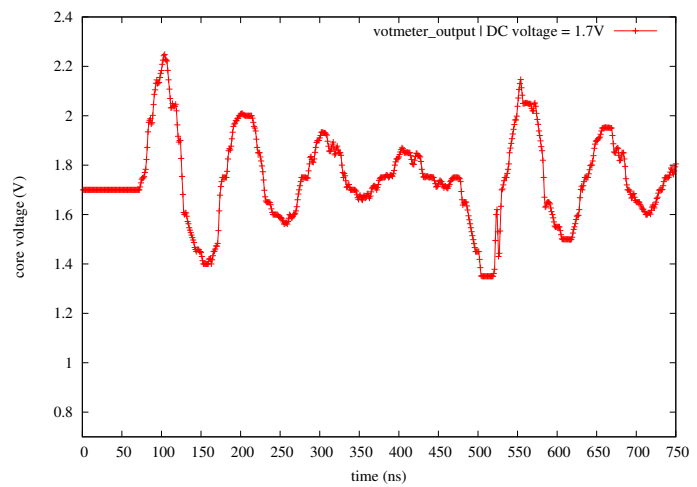

Fig. 6. Core voltage of the FPGA for a (400ns, $+14 \mathrm{~V})$ power supply glitch.

result of these experiments is that a positive power supply glitch induces negative transient voltage modifications under its DC component. For the experiment reported in Fig. 6, the second oscillation (corresponding to the rising edge of the pulse) has a voltage at its tip end which is $300 \mathrm{mV}$ under its DC component. Moreover, this transient underpowering has a duration around 20ns at its tip, which seems well suited to target the rounds of our AES setup.

From the observation of Fig. 6, we drew the counterintuitive assumption that a positive power supply glitch may create setup time constraint violations because of the induced negative oscillations. The following experiments were meant to ascertain this assumption. 
2) Fault injection: Fault injection experiments with a positive power supply glitch $(400 \mathrm{~ns},+14 \mathrm{~V})$ were then carried out on the AES setup. The injection time of the voltage pulse was varied in order to sweep the whole AES' rounds with the tip of the second oscillation which is negative. For each time location, the DC component of the glitch was gradually decreased from $1.7 \mathrm{~V}$ until the injection of the first faults. Following this methodology we succeeded in injecting faults into the various rounds of the AES' calculations. For a given dataset (i.e. the plaintext and key used for the AES encryption), the injected faults were equal to the faults induced with negative power supply glitches. Moreover, the faulted rounds always corresponded to the time location of the negative oscillation tip end. This is an experimental proof that the faults injected with positive power supply glitches are due to setup time violations created by the negative oscillations induced by the voltage pulse edges.

\section{Further results}

According to the previous experiments the pulse width value had no significant effect on the induced voltage perturbations. The main parameters influencing fault injection were the pulse amplitude and DC component values. However, with proper width settings, the oscillation sets will overlap creating offsetting, addition, or sharping effects which may facilitate FI.

1) Offsetting: For a negative voltage pulse with a width equal to the period of the damping oscillation sets, an offsetting effect arises. It consists in offsetting the $2^{\text {nd }}$ negative oscillation induced by the falling edge of the pulse with the $1^{s t}$ positive oscillation of its rising edge. Offsetting allows us to avoid injecting extra faults during the erased oscillation. Fig. 7(a) displays the obtained core voltage perturbations for a $(100 \mathrm{~ns},-14 \mathrm{~V})$ pulse.

2) Addition: The addition effect of a $(50 \mathrm{~ns},+8 \mathrm{~V})$ positive voltage glitch is given in Fig. 7(b), where the 50ns width corresponds to the oscillations half-period. As a result, the amplitude of the $1^{\text {st }}$ negative oscillation is increased by addition of the $1^{\text {st }}$ negative oscillation of the $2^{\text {nd }}$ set: a $400 \mathrm{mV}$ amplitude is obtained for an $8 \mathrm{~V}$ pulse amplitude whereas a $14 \mathrm{~V}$ amplitude was required without an addition effect (see Fig. 7(a).

3) Sharping: Fig. 7(c) illustrates the sharping effect for a $(10 \mathrm{~ns},-22 \mathrm{~V})$ pulse. It consists in a partial overlap of the $1^{\text {st }}$ oscillation induced by the glitch falling and rising edges in order to thin down the faulting negative oscillation. The sharping effect increases the resolution of FI at the expense of its amplitude: the width of the oscillation tip end is decreased to approximately 10ns, while an increase of the pulse amplitude to $22 \mathrm{~V}$ was required to obtain an oscillation amplitude of $400 \mathrm{mV}$ (as obtained in Fig. 7(a) and 7(b) for pulse amplitude of $14 \mathrm{~V}$ and $8 \mathrm{~V}$ resp.).

4) Fault injection: The $(100 \mathrm{~ns},-14 \mathrm{~V}),(50 \mathrm{~ns},+8 \mathrm{~V})$ and $(10 \mathrm{~ns},-22 \mathrm{~V})$ pulses used to illustrate the offsetting, addition and sharping effects were then applied to the AES setup for FI characterization (the same data were used during the following experiments). All AES' rounds were targeted by sweeping the time of the negative oscillation tip end over a large time window. For each time step, an increasing stress was applied to successive encryptions by decreasing step by step the DC component of the voltage glitch from $1.7 \mathrm{~V}$ until a first fault appears. It made it possible to draw a time map of the fault sensitivity threshold of the AES rounds as displayed in Fig. 8(a), 8(b) and 8(c) (the fault sensitivity threshold is expressed as the pulse voltage DC component maximal value inducing faults). The obtained voltage sensitivity shapes are very similar, which was expected because the faulting oscillations have a same oscillation amplitude of $400 \mathrm{mV}$. They differ in time because their respective oscillation tips have different timing w.r.t. the synchronization signal.

The sharped oscillation of Fig. 7(a) achieved the best time resolution: single-bit faults were injected into the whole AES' rounds (except the $1^{\text {st }}$ one). Whereas the broader negative oscillations induces by the (100ns, $-14 \mathrm{~V})$ and $(50 \mathrm{~ns},+8 \mathrm{~V})$ pulses did not succeed in faulting the $6^{\text {th }}$ round (the time sensitivity windows of the $5^{\text {th }}$ and $7^{\text {th }}$ rounds were accordingly expanded as depicted in Fig 8(a) and 8(b).

During these three sets of experiments the faults injected in the same AES round were identical. This proves again that the FI mechanism related to both negative and positive power supply glitches is the same.

\section{CONCLUSION}

In this paper we presented an on-chip delay-based voltmeter. This voltmeter was used to monitor the FPGA core voltage when exposed to power supply glitches. It revealed the induction of two damping oscillation sets inside the target corresponding to the falling and rising edges of the voltage glitch. It also permits us to correlate FI with the tip ends of negative oscillations. Moreover, because the faults injected with negative and positive voltage glitches were identical (and because the FI mechanism of negative voltage glitches was already established to be linked to setup time violations), it demonstrates on a practical basis that the FI mechanism of positive voltage glitches is related to setup time violations. Various techniques of glitch shaping were also introduced that may enhance the efficiency of FI. Designers of secure devices shall be aware that such voltage glitches make it possible to inject single-bit faults with very good timing accuracy.

One interesting consequence of this finding, is that the counter-measures designed to thwart negative power supply glitches should also be effective to defeat FI attempts with positive supply glitches. This will be investigated through further research work.

\section{REFERENCES}

[1] N. Selmane, S. Bhasin, S. Guilley, and J. Danger, "Security evaluation of application-specific integrated circuits and field programmable gate arrays against setup time violation attacks," Information Security, IET, vol. 5, no. 4, pp. 181-190, 2011.

[2] L. Zussa, J.-M. Dutertre, J. Clédière, B. Robisson, and A. Tria, "Investigation of timing constraints violation as a fault injection means," DCIS'2012: XVII Conference on Design of Circuits and Integrated Systems, 2012. 


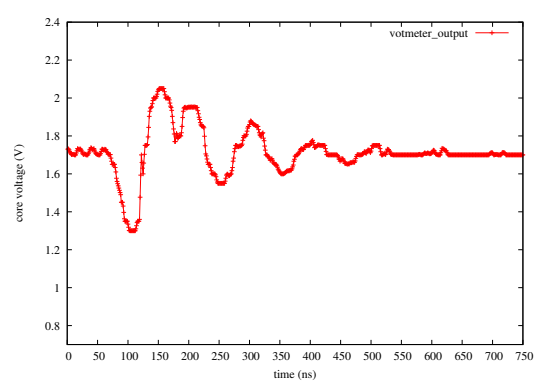

(a) $(100 \mathrm{~ns},-14 \mathrm{~V})$

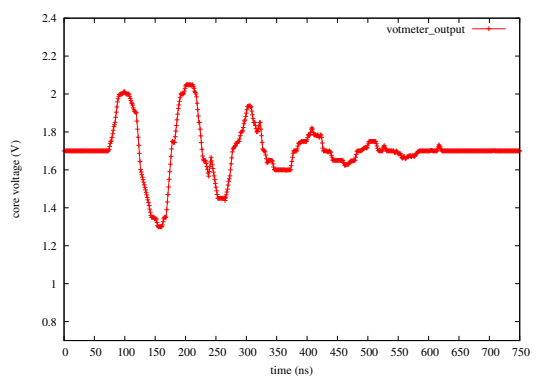

(b) $(50 \mathrm{~ns},+8 \mathrm{~V})$

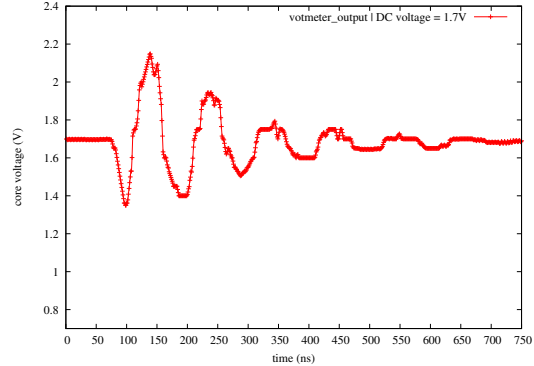

(c) $(10 \mathrm{~ns},-22 \mathrm{~V})$

Fig. 7. Core voltage curves of the FPGA for various pulse settings.

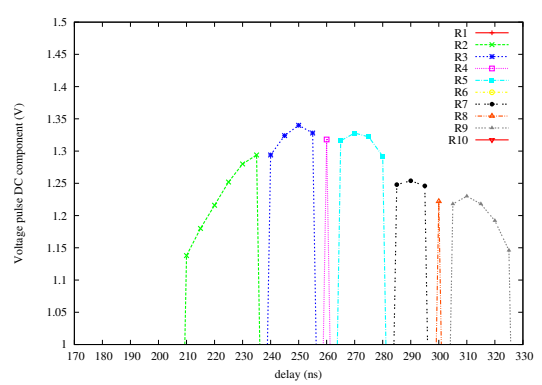

(a) $(100 \mathrm{~ns},-14 \mathrm{~V})$

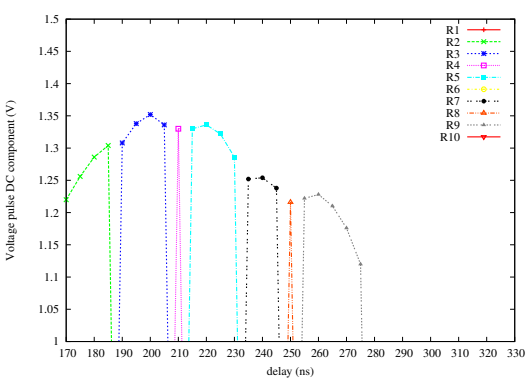

(b) $(50 \mathrm{~ns},+8 \mathrm{~V})$

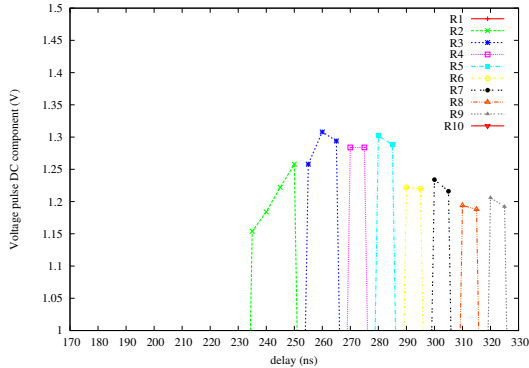

(c) $(10 \mathrm{~ns},-22 \mathrm{~V})$

Fig. 8. Faulted AES' rounds for various pulse DC component and time settings.

[3] M. Hutter, J.-M. Schmidt, and T. Plos, "Contact-based fault injections and power analysis on rfid tags," in Circuit Theory and Design, 2009. ECCTD 2009. European Conference on. IEEE, 2009, pp. 409-412.

[4] K. M. Zick, M. Srivastav, W. Zhang, and M. French, "Sensing nanosecond-scale voltage attacks and natural transients in fpgas," in Proceedings of the ACM/SIGDA international symposium on Field programmable gate arrays. ACM, 2013, pp. 101-104.

[5] K. A. Bowman, C. Tokunaga, J. W. Tschanz, A. Raychowdhury, M. M. Khellah, B. M. Geuskens, S.-L. Lu, P. A. Aseron, T. Karnik, and V. K. De, "All-digital circuit-level dynamic variation monitor for silicon debug and adaptive clock control," Circuits and Systems I: Regular Papers, IEEE Transactions on, vol. 58, no. 9, pp. 2017-2025, 2011.

[6] NIST, "Announcing the advanced encryption standard (aes)," Federal Information Processing Standards Publication 197, 2001.

[7] J. M. Rabaey, A. Chandrakasan, and B. Nikolic, Digital Integrated Circuits. Prentice Hall, 2003.

[8] A. Barenghi, G. Bertoni, L. Breveglieri, M. Pellicioli, and G. Pelosi, "Low voltage fault attacks to aes," in HOST, 2010, pp. 7-12.

[9] A. Barenghi, L. Breveglieri, I. Koren, and D. Naccache, "Fault injection attacks on cryptographic devices: Theory, practice, and countermeasures." in Proceedings of the IEEE, 2012.

[10] P. Dudek, S. Szczepanski, and J. V. Hatfield, "A high-resolution cmos time-to-digital converter utilizing a vernier delay line," Solid-State Circuits, IEEE Journal of, vol. 35, no. 2, pp. 240-247, 2000. 